\title{
Relationship between serum cardiac troponin $T$ level and cardiopulmonary function in stable chronic obstructive pulmonary disease
}

This article was published in the following Dove Press journal:

International Journal of COPD

10 February 2015

Number of times this article has been viewed

\section{Kumiko Hattori \\ Takeo Ishii \\ Takashi Motegi \\ Yuji Kusunoki \\ Akihiko Gemma \\ Kozui Kida}

Department of Pulmonary Medicine and Oncology; Respiratory Care Clinic, Nippon Medical School, Tokyo, Japan
Correspondence: Kozui Kida Department of Pulmonary Medicine, Infection, and Oncology, Respiratory Care Clinic, Nippon Medical School, 4-7I5-8F, Kudan-minami, Chiyoda-ku, Tokyo 102-0074, Japan

Tel +8I 352762325

Fax +81352762326

Email kkida@nms.ac.jp
Background: High-sensitivity cardiac troponin T (hs-cTnT) in serum is a useful marker of acute myocardial injury, yet information is limited in patients with chronic obstructive pulmonary disease. We aimed to explore the association between hs-cTnT levels and cardiac and pulmonary dysfunction in patients with stable chronic obstructive pulmonary disease and at-risk individuals.

Methods: We examined community-dwelling adults with/without chronic obstructive pulmonary disease, with a life-long smoking history, current symptoms of dyspnea during exertion, prolonged coughing, and/or sputum. Serum hs-cTnT concentrations were measured, and subjects underwent pulmonary function tests, high-resolution computed tomography of the chest, an echocardiogram, and a 6-minute walking test.

Results: Eighty-six stable patients were identified (mean age 65.5 years; predicted forced expiratory volume in 1 second $\left[\mathrm{FEV}_{1} \%\right.$ predicted] $75.0 \%$ ). Their overall mean hs-cTnT level was $0.008 \mathrm{ng} / \mathrm{mL}$. Logarithmically transformed hs-cTnT levels significantly and positively correlated with age, smoking index, serum high-sensitivity $\mathrm{C}$-reactive protein levels, right ventricle systolic pressure, low attenuation area percentage, and brain natriuretic peptide levels (range $r=0.231-0.534, P=0.000$ to $P=0.042$ ). Further, logarithmically transformed hs-cTnT values significantly and negatively correlated with forced vital capacity, $\mathrm{FEV}_{1} \%$ predicted, diffusion capacity, arterial oxygen tension, and 6-minute walking distance (range $r=-0.482$ to -0.377 , $P=0.000$ to $P=0.002$ ). Multivariate analyses showed that hs-cTnT values varied independently according to the following three parameters: high-sensitivity $C$-reactive protein levels $(B=0.157$, $\beta=0.450, t=3.571, P=0.001)$, age $(B=0.008, \beta=0.352, t=2.789, P=0.009)$, and right ventricular systolic pressure $(B=0.008, \beta=0.280, t=2.202, P=0.035)$.

Conclusion: Even in patients with stable chronic obstructive pulmonary disease, the serum troponin $\mathrm{T}$ concentration was controlled by at least three major factors, ie, systemic inflammation, advancing age, and right cardiac overload.

Keywords: chronic obstructive pulmonary disease, troponin T, C-reactive protein, aging

\section{Introduction}

Previous studies have indicated that ischemic heart disease is a major cause of death in patients with chronic obstructive pulmonary disease (COPD), ${ }^{1,2}$ suggesting that acute myocardial infarction should be differentiated from or identified in coexistence with an acute exacerbation of COPD. Since clinical symptoms of acute exacerbation of COPD and acute myocardial infarction are often similar, differential diagnosis may be difficult.

Cardiac injury is defined as disruption of the normal cardiac myocyte membrane integrity, resulting in loss of its intracellular contents into the extracellular space (such 
as blood). This loss results in detectable levels of a variety of biologically active cytosolic and structural proteins in the blood, including troponins, creatine kinase, myoglobin, hearttype fatty acid-binding proteins, and lactate dehydrogenase. ${ }^{3}$ Therefore, serum troponin $\mathrm{T}$ ( $\mathrm{TnT})$, released with cardiac stress, ${ }^{4}$ is considered a useful biomarker for cardiac injury. A study using pacing and blood sampling from the coronary sinus and peripheral blood showed that high-sensitivity cardiac troponin $\mathrm{T}$ (hs-cTnT) increases in both the coronary sinus and in peripheral blood, regardless of whether lactate levels in the coronary sinus are elevated. ${ }^{5}$ These findings could suggest that hs-cTnT is released because of cardiac stress and repeated injury. Alternatively, another hypothesis purports that the elevations could represent partially reversible myocyte injury. Other proposed sources of troponin release include apoptosis, normal myocyte turnover, cellular release of proteolytic troponin breakdown products, increased cell wall permeability, and formation and release of membranous blebs. ${ }^{6}$ Available data suggest that hs-cTnT increases in acute exacerbation ${ }^{7-10}$ and even in stable COPD. ${ }^{11}$ Other data based on necropsy findings show that left ventricular hypertrophy occurs in 10\%-90\% of COPD cases. ${ }^{12}$ This finding strongly suggests that left ventricular hypertrophy and dysfunction frequently occur in COPD. Several potential mechanisms have been proposed for left ventricular failure in COPD. Although hs-cTnT has been proven to be a useful biomarker for differential diagnosis in patients hospitalized with a severe COPD exacerbation, ${ }^{13}$ it is not clear whether hs-cTnT levels change in patients with stable COPD. In this study, we explored the association between hs-cTnT levels and cardiac and pulmonary dysfunction in patients with stable COPD and in individuals at risk of COPD, in order to elucidate the meaning of hs-cTnT values in patients with stable COPD in a clinical setting.

\section{Materials and methods}

\section{Study population}

This prospective study examined 548 subjects with a lifelong history of smoking and current symptoms of dyspnea during exertion, prolonged coughing, and/or sputum. Patients with any cardiovascular disease $(n=232)$ or other respiratory disease $(n=129)$, such as bronchiectasis or lung fibrosis, were carefully excluded as mentioned below, and 187 subjects with COPD-related symptoms were examined. The study was conducted in accordance with the Declaration of Helsinki and the International Conference on Harmonisation Tripartite Guideline for Good Clinical Practice. Before the studies were initiated, the protocol was reviewed and approved by the ethics committee of the Nippon Medical School (approval number 18-11-31). All patients were required to provide written, informed consent before enrollment. Finally, 86 subjects were enrolled with informed consent in the present study, and they were also confirmed to have been in a stable condition for at least 3 months.

\section{Exclusion criteria}

Subjects suffering from any one of the following pulmonary and cardiovascular diseases were excluded: bronchial asthma, bronchiectasis, interstitial pneumonia, hypertension, ischemic heart disease, any type of arrhythmia, and congestive heart failure. Detailed information is provided in Figure $\mathrm{S} 1$.

\section{Measurements}

All subjects underwent chest roentgenography from both directions, routine blood chemistry, electrocardiogram, pulmonary function tests, blood gas analysis, echocardiogram, 6-minute walking test (6MWT), and high-resolution computed tomography (HRCT) of the chest. An electrocardiogram was performed to identify obvious heart disease such as arrhythmia and previous myocardial infarction. Serum samples were taken simultaneously and were preserved at $-80^{\circ} \mathrm{C}$ for hs-cTnT measurements.

\section{Pulmonary function tests}

The pulmonary tests were performed by well-trained technicians according to the American Thoracic Society guidelines, ${ }^{14}$ using specialized equipment for lung function testing with computer processing (Chestac 55, Chest Co., Tokyo, Japan). Subjects were diagnosed with COPD according to a value of $<0.70$ for the post-bronchodilator forced expiratory volume in 1 second $\left(\mathrm{FEV}_{1}\right)$ /forced vital capacity (FVC) ratio, as indicated by the Global Initiative for Chronic Obstructive Lung Disease. ${ }^{15}$ Subjects not meeting the COPD criteria were classified into the "at-risk" group.

\section{HRCT parameters}

We performed helical HRCT scans (details in Supplementary materials). Percentage of low attenuation area (LAA\%) for the upper, middle, and lower zones of bilateral lung fields and overall mean values were calculated as previously reported. ${ }^{16}$

\section{Echocardiography}

Echocardiography was performed using a diagnostic ultrasound system (Aplio SSA-770A, Toshiba Medical Systems Co., Tokyo, Japan; details in Supplementary materials). 


\section{Serum troponin measurement}

Serum hs-cTnT levels were measured using an electrochemiluminescence immunoassay (BML, Inc., Kawagoe, Japan). The lower limit of detection for hs-cTnT was $0.003 \mathrm{ng} / \mathrm{mL}$, and the standard value was below $0.014 \mathrm{ng} / \mathrm{mL}$.

\section{Statistical analysis}

The following parameters were not normally distributed and were therefore transformed logarithmically: hs-cTnT concentration, $\mathrm{FEV}_{1} \%$ predicted, percent diffusing capacity of the lung for carbon monoxide/alveolar volume (\%DLco/ VA), high-sensitivity C-reactive protein (hs-CRP), and walking distance in the 6MWT. The associations between hs-cTnT levels and other parameters were tested using Pearson's correlation analyses and single linear regression analysis. Among the parameters, the following showed significant differences by Pearson's analysis: age, smoking habit, $\mathrm{FVC}, \mathrm{FEV}_{1} \%$ predicted, \%DLco/VA, partial pressure of oxygen $\left(\mathrm{P}_{\mathrm{a}} \mathrm{O}_{2}\right)$, serum hs-CRP level, right ventricular systolic pressure (RVSP), walking distance, and LAA $\%$ for the upper and lower lung fields. Therefore, we performed multivariate, stepwise backward analyses for hs-cTnT serum concentrations.

The data were analyzed using PASW Statistics version 18 for Windows software (SPSS Inc, Chicago, IL, USA). All $P$-values were two-tailed, and a $P$-value of $<0.05$ was considered to be statistically significant.

\section{Results}

The study design is shown in Figure 1. Among the total of 548 subjects, 86 were selected as the patient group who either had or were at risk of COPD. In total, 361 subjects were excluded after various examinations because of either cardiac comorbidities or other pulmonary diseases (Figures 1 and S1).

The basic characteristics of the subjects are shown in Table 1. The mean age was 65.5 years. Based on the COPD criteria, 54 subjects were diagnosed with COPD, 32 subjects were diagnosed as being at risk of COPD, and the mean $\mathrm{FEV}_{1} \%$ predicted was $75.0 \%$. The numbers of patients for each Global Initiative for Chronic Obstructive Lung Disease $(2004)^{15}$ classification were as follows: $13,22,17$, and 2

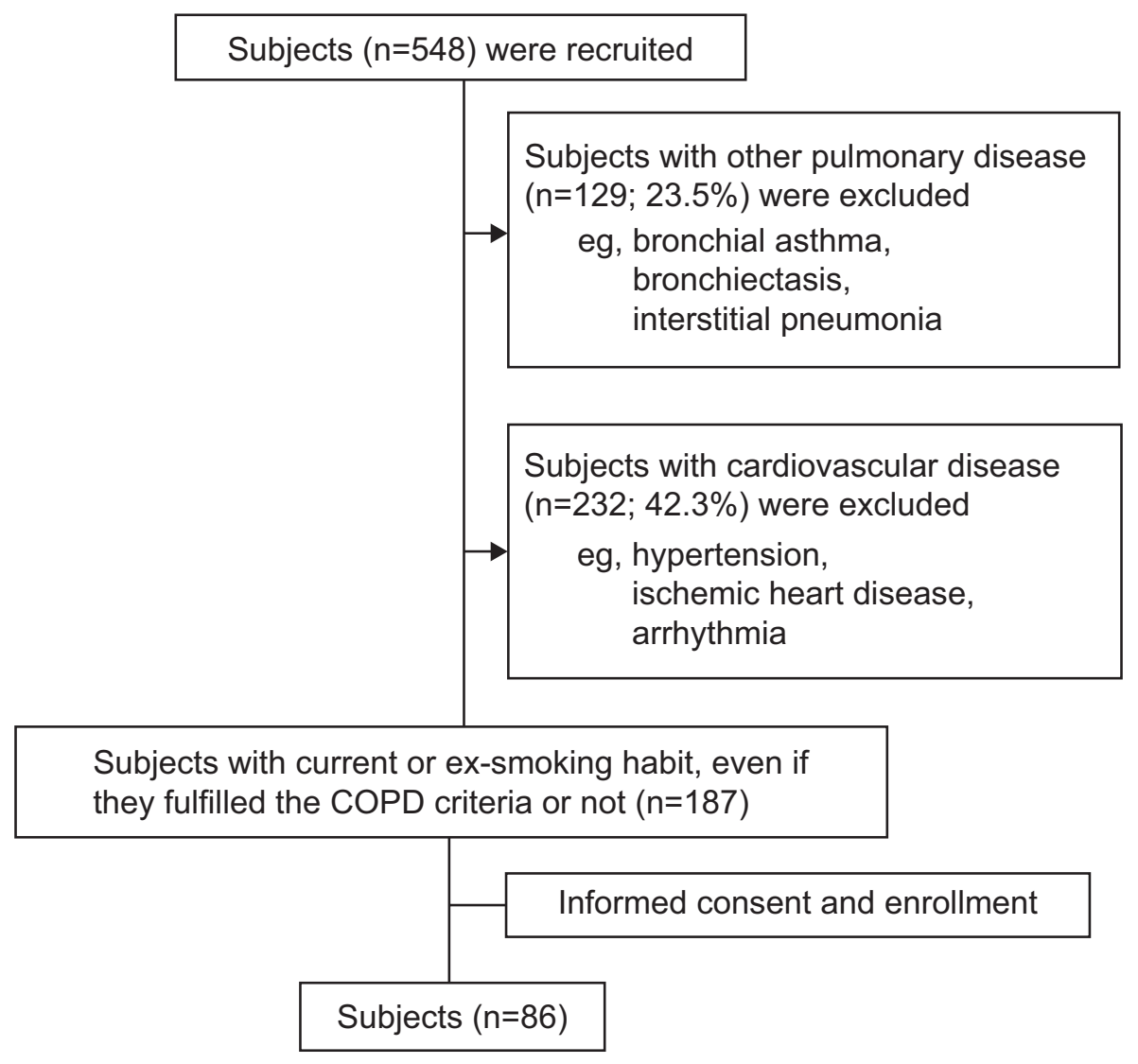

Figure I Study flow chart. Subjects ( $\mathrm{n}=548$ ) were recruited, and subjects with other pulmonary disease and/or any cardiovascular disease were excluded (see Supplementary materials). A total of 86 subjects were enrolled in the study.

Abbreviation: COPD, chronic obstructive pulmonary disease. 
Table I Baseline patient characteristics

\begin{tabular}{|c|c|}
\hline & Subjects $(n=86)$ \\
\hline Age (years) & $65.5 \pm 11.3$ \\
\hline Sex (male/female) & $79 / 7$ \\
\hline Smoking habit (pack/year) & $70.4 \pm 43.4$ \\
\hline BMI & $21.6 \pm 2.6$ \\
\hline $\mathrm{FEV}_{1}(\mathrm{~L})$ & $2.2 \pm 1.0$ \\
\hline $\mathrm{FVC}(\mathrm{L})$ & $3.5 \pm 0.9$ \\
\hline $\mathrm{FEV}, \%(\%)$ & $60.7 \pm 17.1$ \\
\hline FEV $\%$ predicted (\%) & $75.0 \pm 26.0$ \\
\hline \% DLco/VA (\%) & $69.6 \pm 22.1$ \\
\hline Diagnosis (COPD/at risk) & $54 / 32$ \\
\hline $\mathrm{P}_{\mathrm{a}} \mathrm{O}_{2}$ (Torr) & $82.2 \pm 13.4$ \\
\hline WBC $\left(10^{9} / \mathrm{L}\right)$ & $6.2 \pm 2.0$ \\
\hline Neutrophils $\left(10^{9} / \mathrm{L}\right)$ & $3.8 \pm 1.9$ \\
\hline Hemoglobin (g/dL) & $\mid 4.1 \pm 1.5$ \\
\hline Creatinine $(\mathrm{mg} / \mathrm{dL})$ & $0.75 \pm 0.13$ \\
\hline $\mathrm{BNP}(\mathrm{pg} / \mathrm{mL})$ & $25.5 \pm 32.9$ \\
\hline hs-CRP (mg/dL) & $0.182 \pm 0.343$ \\
\hline hs-cTnT (ng/mL) & $0.008 \pm 0.005$ \\
\hline \multicolumn{2}{|c|}{$\begin{array}{l}\text { Abbreviations: } \mathrm{BMI} \text {, body mass index; } \mathrm{FEV}_{1} \text {, forced expiratory volume in } \\
\text { I second; FVC, forced vital capacity; DLco/VA, diffusing capacity of the lung for } \\
\text { carbon monoxide/alveolar volume; COPD, chronic obstructive pulmonary disease; } \\
\mathrm{P}_{\mathrm{a}} \mathrm{O}_{2} \text {, partial pressure of oxygen; WBC, white blood cells; BNP, brain natriuretic } \\
\text { peptide; hs-CRP, high sensitivity C-reactive protein; hs-cTnT, high sensitivity cardiac } \\
\text { troponin T. }\end{array}$} \\
\hline
\end{tabular}

for class I, II, III, and IV, respectively. Mean hs-CRP and hs-cTnT serum levels were $0.182 \mathrm{mg} / \mathrm{dL}$ and $0.008 \mathrm{ng} / \mathrm{mL}$, respectively. Eight patients had hs-cTnT levels below the level of detection $(0.003 \mathrm{ng} / \mathrm{mL})$. In addition, hs-cTnT levels in seven subjects with COPD and in one subject in the at-risk group were over the normal limit of 0.014 (range $0.016-0.027) \mathrm{mg} / \mathrm{mL}$.

The results of chest HRCT, echocardiography, and the 6MWT are shown in Table 2. The percentage of subjects with emphysema was $71.4 \%$ (all, $\mathrm{n}=60$; COPD, $\mathrm{n}=51$; at-risk, $\mathrm{n}=9$ ). Mean RVSP was $33.7 \mathrm{mmHg}$. The 6MWT revealed 21 subjects with hypoxia, whose mean minimum oxygen saturation was $82.5 \%$ (range $71 \%-89 \%$ ), and whose mean delta saturation of pulse oximetry oxygen $\left(\Delta \mathrm{SpO}_{2}\right.$; defined as the difference between the initial value at rest and the minimum value during the test) was $10.4 \%$ (range 6\%-24\%).

The correlations between logarithmically transformed hs-cTnT levels and demographic data, results of the various pulmonary function tests, arterial blood gas, and other laboratory data are shown in Table 3. The hs-cTnT level (logged) was significantly and positively correlated with age $(r=0.534$, $P=0.000)$, smoking habit $(r=0.280, P=0.042)$, brain natriuretic peptide ( $r=0.334, P=0.004)$, hs-CRP (logged; $r=0.517$, $P=0.000)$, RVSP $(r=0.008, P=0.317)$, and LAA\% for the upper $(r=0.231, P=0.042)$ and lower $(r=0.286, P=0.011)$
Table 2 Results of HRCT, echocardiography, and 6-minute walking test

\begin{tabular}{ll}
\hline & Subjects $(\mathbf{n}=\mathbf{8 6})$ \\
\hline Chest HRCT & \\
LAA\% for the upper lung field $(\%)$ & $30.0 \pm 17.1$ \\
LAA\% for the lower lung field $(\%)$ & $28.5 \pm 15.1$ \\
Echocardiography & \\
HR (beats/minute) & $74.9 \pm 12.0$ \\
IVS Td (mm) & $8.8 \pm 1.1$ \\
PW Td (mm) & $8.9 \pm 1.0$ \\
LAD (mm) & $31.4 \pm 5.3$ \\
LVEDV (mL) & $101.4 \pm 24.7$ \\
EF $(\%)$ & $68.1 \pm 5.5$ \\
SV (mL) & $69.5 \pm 16.9$ \\
CO (L/minute) & $5.2 \pm 1.4$ \\
RVSP (mmHg) & $33.7 \pm 6.3$ \\
IVC Ex (mm) & $12.9 \pm 2.7$ \\
Six-minute walking test & \\
Walking distance $(\mathrm{m})$ & $517.5 \pm 96.5$ \\
$\Delta S p O_{2}(\%)$ & $5.2 \pm 5.4$ \\
\hline
\end{tabular}

Abbreviations: LAA, low attenuation area; HRCT, high-resolution computed tomography; HR, heart rate; IVS Td, thickness of interventricular septum in diastole; PW Td, thickness of posterior wall in diastole; LAD, left atrial dimension; LVEDV, left ventricular end-diastolic volume; $\mathrm{EF}$, ejection fraction; SV, stroke volume; $\mathrm{CO}$, cardiac output; RVSP, right ventricular systolic pressure; IVC Ex, diameter of inferior vena cava in expiration; $\Delta \mathrm{SpO}_{2}$, delta saturation of pulse oximetry oxygen.

lung fields. On the other hand, there was significant and negative correlation with $\mathrm{FVC}(r=-0.388, P=0.000), \mathrm{FEV}_{1} \%$ predicted (logged; $r=-0.377, P=0.001$ ), \%DLco/VA (logged; $r=-0.431, P=0.000), \mathrm{P}_{\mathrm{a}} \mathrm{O}_{2}(r=-0.385, P=0.002)$, and walking distance (logged; $r=-0.482, P=0.000$ ).

The associations between hs-cTnT levels (logged) and demographic and cardiopulmonary parameters in a linear regression analysis model are shown in Figure 2. Age was the parameter best fitting to hs-cTnT values (logged; Figure 2A).

To determine the variables affecting the hs-cTnT values (logged), multiple regression analysis with a stepwise estimation model was used on parameters that appeared to be significantly correlated with hs-cTnT values (logged) in Pearson's correlation analyses. Multivariate analysis revealed that hs-cTnT levels were independently affected by the following three parameters: hs-CRP (logged; regression coefficient $B=0.157$, standardized regression coefficient $\beta=0.450, t=3.571$, $P=0.001)$, age $(B=0.008, \beta=0.352, t=2.789, P=0.009)$, and $\operatorname{RVSP}(B=0.008, \beta=0.280, t=2.202, P=0.035)$. There was no multicollinearity among these variables. Thus, hs-CRP values (logged), age, and RVSP were independent variables affecting hs-cTnT values (logged; $R^{2}=0.506$; Table 4 ). We also checked whether this multivariate model for hs-cTnT would be accurate for both a subgroup of COPD and at-risk subjects. Hs-CRP 
Table 3 Association between hs-cTnT levels (logged) and demographic and cardiopulmonary parameters in all subjects

\begin{tabular}{|c|c|c|}
\hline & \multicolumn{2}{|c|}{ Subjects $(n=86)$} \\
\hline & $r$ & $P$-value \\
\hline Age & 0.534 & 0.000 \\
\hline Smoking habit & 0.280 & 0.042 \\
\hline BMI & 0.075 & 0.515 \\
\hline FVC & -0.388 & 0.000 \\
\hline $\mathrm{FEV}_{1} \%$ predicted (logged) & -0.377 & 0.001 \\
\hline \% DLco/VA (logged) & -0.431 & 0.000 \\
\hline $\mathrm{P}_{\mathrm{a}} \mathrm{O}_{2}$ & -0.385 & 0.002 \\
\hline WBC & 0.212 & 0.062 \\
\hline BNP & 0.334 & 0.004 \\
\hline hs-CRP (logged) & 0.517 & 0.000 \\
\hline IVS Td & 0.170 & 0.137 \\
\hline PW Td & 0.173 & 0.131 \\
\hline LAD & 0.112 & 0.333 \\
\hline LVEDV & -0.020 & 0.865 \\
\hline EF & 0.058 & 0.612 \\
\hline $\mathrm{CO}$ & 0.201 & 0.082 \\
\hline RVSP & 0.317 & 0.008 \\
\hline Walking distance (logged) & -0.482 & 0.000 \\
\hline$\Delta \mathrm{SpO}_{2}$ & 0.206 & $0.07 I$ \\
\hline LAA\% for the upper lung field & 0.231 & 0.042 \\
\hline LAA\% for the lower lung field & 0.286 & 0.011 \\
\hline
\end{tabular}

Abbreviations: BMI, body mass index; FVC, forced vital capacity; FEV, forced expiratory volume in I second; DLco/VA, diffusing capacity of the lung for carbon monoxide/alveolar volume; $\mathrm{P}_{2} \mathrm{O}_{2}$, partial pressure of oxygen; $\mathrm{WBC}$, white blood cells; BNP, brain natriuretic peptide; hs-CRP, high-sensitivity C-reactive protein; IVS Td, thickness of interventricular septum in diastole; PW Td, thickness of posterior wall in diastole; LAD, left atrial dimension; LVEDV, left ventricular end-diastolic volume; EF ejection fraction; $\mathrm{CO}$, cardiac output; RVSP, right ventricular systolic pressure; $\triangle \mathrm{SpO}_{2}$, delta saturation of pulse oximetry oxygen; LAA, low attenuation area; hs-cTnT, highsensitivity cardiac troponin $\mathrm{T}$.

and age, but not RVSP, were associated with hs-cTnT in this model for these two subgroups (Tables S1 and S2).

The hs-cTnT level was low in the entire population. Thus, we also performed simple correlation analyses between hs-cTnT and clinical parameters (Table S3); further, we performed stepwise regression analysis on logged hs-cTnT values (Table S4) again in the subjects whose hs-cTnT level was greater than the median (0.007). Although the population was relatively small in this analysis, we were able to observe an association between hs-cTnT and hs-CRP.

\section{Discussion}

In the present study, we have shown that hs-cTnT in the serum of patients with stable COPD and in at-risk subjects can be explained by three independent factors, ie, hs-CRP, age, and RVSP. In addition to strict selection criteria aimed at including only COPD patients and exclude those with any cardiac or pulmonary comorbidities, all subjects were confirmed to have been in a stable condition for at least 3 months prior to the study.
We used the fixed $\mathrm{FEV}_{1} / \mathrm{FVC}$ ratio to define airflow obstruction since the use of $\mathrm{FEV}_{1} \%$ predicted is commonly used in clinical practice; however, using $\mathrm{FEV}_{1} \%$ predicted might result in overdiagnosis of COPD in elderly individuals. ${ }^{17}$ Therefore, from the onset of the study, we recruited patients who had a life-long history of smoking and continuous respiratory symptoms, such as cough, expectoration, and/or dyspnea in daily exercise. These patients were then assigned to one of two groups, ie, COPD or at-risk. ${ }^{15}$ Interestingly, $42.3 \%$ of the original 548 subjects who were recruited into this study and had COPD-related symptoms also had some cardiovascular comorbidity. Currently, evidence regarding the role of hs-cTnT in subjects with COPD is of high interest; hs-cTnT levels increase in acute exacerbation of COPD, and elevation of hs-cTnT levels is associated with a poor prognosis. ${ }^{7-10} \mathrm{An}$ increased hs-cTnT concentration was observed among some patients with stable COPD, and was significantly and positively associated with serum interleukin- 6 concentration and the presence of pathologic $\mathrm{Q}$ waves, and negatively associated with $\mathrm{FEV}_{1}{ }^{1{ }^{11}}$ The reason for the increase in hs-cTnT levels in stable COPD is still subject to speculation and is the subject of several hypotheses described herein.

First, Neukamm et $\mathrm{al}^{11}$ hypothesized that the hs-cTnT increase is induced by systemic inflammation, which is common in the pathogenesis of COPD, cardiovascular diseases, and hypoxemia. Consistent with this hypothesis, they reported that higher hs-TnT levels are associated with higher interleukin-6 concentrations, which are thought to reflect systemic inflammation. ${ }^{11}$ Here, we have further demonstrated that hs-cTnT is closely associated with hs-CRP, reflective of systemic inflammation. The previously reported association between increased hs-cTnT and decreased $\mathrm{FEV}_{1}{ }_{1}^{11}$ was also observed in this study. Moreover, our data demonstrate a close association between increased hs-cTnT and decreased $\mathrm{FVC}, \mathrm{FEV}_{1} \%$ predicted, and \%DLco/VA. Thus, local inflammation in the lungs of COPD patients might be transmitted to the entire body, including the heart, ${ }^{18}$ and induce cardiomyopathy. If a medication, eg, an inhaled corticosteroid, could reduce systemic inflammation, ${ }^{19}$ it could also inhibit occurrence of cardiac comorbidities in COPD subjects with high hs-cTnT values.

Second, it was interesting to observe that reduced $\mathrm{P}_{\mathrm{a}} \mathrm{O}_{2}$ was associated with increased hs-cTnT (logged; $r=-0.38$, $P=0.002$ ). Since higher hs-cTnT was associated with more severe airflow obstruction and hypoxemia in COPD subjects without cardiac comorbidities, patients with more severe COPD might have latent and progressive cardiomyopathy with a relatively low hs-cTnT value, but higher than in non-COPD 
A

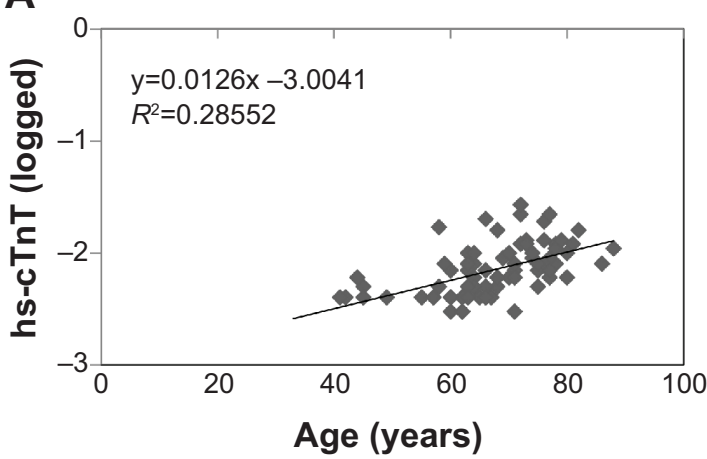

C

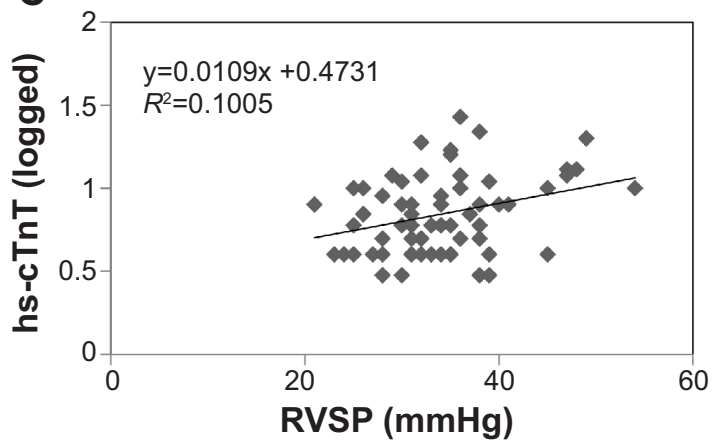

B

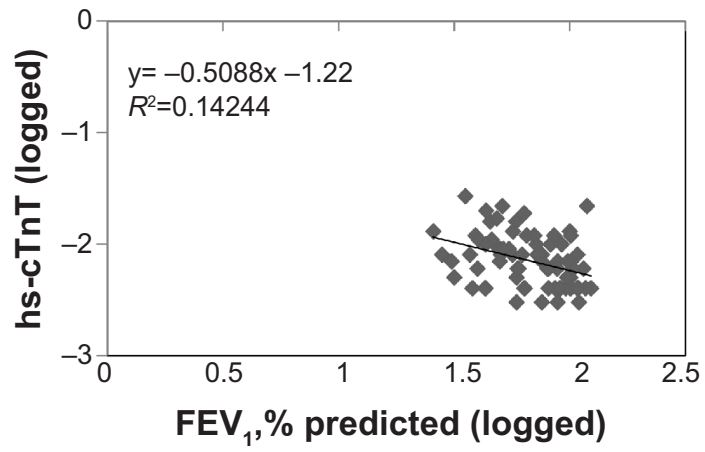

D

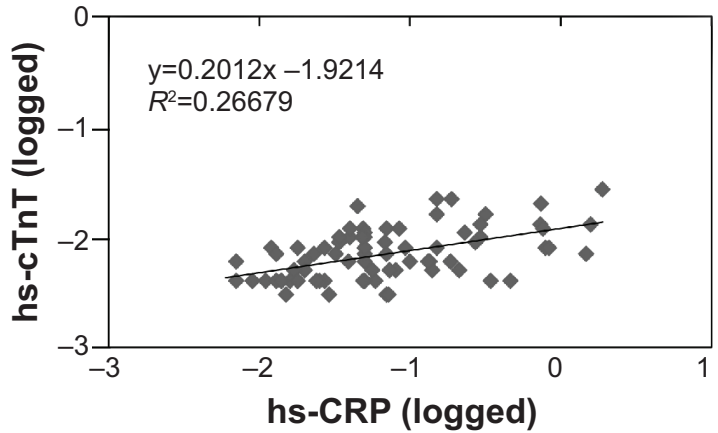

Figure 2 Association between log(hs-cTnT) and demographic and cardiopulmonary parameters in single linear regression analysis. Notes: (A) Age, (B) FEV \% predicted (logged), (C) RVSP, (D) hs-CRP (logged).

Abbreviations: FEV \% predicted, predicted forced expiratory volume in I second; RVSP, right ventricular systolic pressure; hs-CRP, high-sensitivity C-reactive protein; hs-cTNT, high-sensitivity cardiac troponin T.

subjects, possibly due to prolonged hypoxemia. We might be able to predict whether some COPD patients will have cardiac comorbidity in the near future by measuring hs-cTnT and prevent the occurrence by long-term oxygen supplementation. If this hypothesis is correct, the current criteria used for long-term oxygen therapy ${ }^{20}$ should be amended, as they are primarily based on data at rest. In the same vein, attention should be paid to cardiac injury by hypoxemia, which occurs not only at rest but also during exercise. Since we only observed correlation between hs-cTnT in the serum and $\mathrm{P}_{\mathrm{a}} \mathrm{O}_{2}$, a causal relationship between hs-cTnT and $\mathrm{P}_{\mathrm{a}} \mathrm{O}_{2}$ remains to be elucidated.

Third, cardiovascular risk factors, morbidity, and mortality are reportedly associated with increased hs-cTnT levels among the aged population, particularly in men. ${ }^{21,22}$ The mean age of the recruited subjects in this study was 65.5 years, and $91.9 \%$ were men. Multivariate analyses showed that age was one of the independent factors that explained increased hscTnT, consistent with recent data by Van Remoortel et al. ${ }^{23}$ Therefore, COPD is characterized by multi-morbidity, and is independently caused by age, smoking, physical inactivity, systemic inflammation, and obesity. Hence, older male subjects with COPD might be at a higher risk for cardiac injury, as previously observed. ${ }^{21,22}$ Notably, in the present study, only $34.1 \%$ of patients remained after excluding those who had cardiac and/or pulmonary comorbidity. This observation implies that most patients, who might normally be suspected of having COPD or of being at risk, may in fact be suffering from other such comorbidities. This deduction

Table 4 Multiple regression analysis for hs-cTnT levels (logged, stepwise estimation model)

\begin{tabular}{|c|c|c|c|c|c|c|}
\hline Explanatory variable & B & $95 \% \mathrm{Cl}$ & & $\beta$ & t-value & $P$-value \\
\hline (Constant) & -2.797 & -3.271 & -2.322 & & -12.012 & 0.000 \\
\hline hs-CRP (logged) & 0.157 & 0.067 & 0.246 & 0.450 & $3.57 \mid$ & 0.001 \\
\hline Age & 0.008 & 0.002 & 0.014 & 0.352 & 2.789 & 0.009 \\
\hline RVSP & 0.008 & 0.001 & 0.016 & 0.280 & 2.202 & 0.035 \\
\hline
\end{tabular}

Abbreviations: $B$, regression coefficient; $\mathrm{Cl}$, confidence interval; $\beta$, standardized regression coefficient; hs-CRP, high-sensitivity $C$-reactive protein; RVSP, right ventricular systolic pressure; hs-cTnT, high-sensitivity cardiac troponin $\mathrm{T}$. 
generally describes the nature of chronic disease in the elderly, and it is consistent with recent data by Van Remoortel et al. ${ }^{23}$ Interestingly, the results of the present study might suggest that cardiovascular comorbidities do not occur in severe COPD alone and also that cardiac injury occurs in stable or even in mild COPD. In this regard, serum hs-cTnT is a promising biomarker of cardiovascular dysfunction or injury in patients with COPD. However, we have not tested whether hs-cTNT is a sensitive marker for cardiac injury in COPD, and it would be interesting to examine the COPD patients with cardiac injury to investigate this matter in the future. Clearly, this hypothesis should also be tested on a large population with consideration of potential ethnic differences.

Our study has some limitations defined herein. First, there are limitations inherent in the sample size. Before starting this study, we determined that the required sample size was 85 to detect a simple correlation between hs-cTnT and continuous variables related to $\mathrm{COPD}$ (eg, $\mathrm{FEV}_{1} \%$ predicted) with a correlation coefficient $(r)$ of $0.3,80 \%$ power $(\beta=0.2)$, and a significance level of $5 \%(\alpha=0.05){ }^{24}$ Because 86 subjects were included, the sample size was large enough to accurately calculate and test simple correlations. However, we also performed multiple regression analyses, in which the sample size for this type of study could be smaller. Second, all subjects enrolled in this study were recruited from a secondary referral medical clinic. This recruitment did not allow us to evaluate the effects of medications, since most patients had already been administered medication prior to their referral to our clinic. Third, recent reports indicated that hs-cTnT levels were higher in patients with obstructive sleep apnea syndrome (OSAS) and that hs-cTnT is significantly associated with apnea and hypopnea index. ${ }^{25,26}$ Randby et $\mathrm{al}^{26}$ suspect that increased activity of the sympathetic nervous system, increased heart rates, and intermittent increase of blood pressure induce elevated hs-cTnT levels in patients with OSAS. We did not exclude possible OSAS comorbidity by polysomnography; this association requires further investigation in patients with stable COPD. Concomitant COPD and OSAS, termed the overlap syndrome, is not rare and affects at least $1 \%$ of the general population. ${ }^{27}$ Fourth, future studies should examine a non-smoker cohort with COPD.

\section{Conclusion}

In conclusion, the increased hs-cTnT concentration was explained by three independent factors, ie, hs-CRP, age, and right cardiac overload, in patients with stable COPD and at-risk subjects.

\section{Disclosure}

The authors report no conflicts of interest in this work.

\section{References}

1. Patel AR, Hurst JR. Extrapulmonary comorbidities in chronic obstructive pulmonary disease: state of the art. Expert Rev Respir Med. 2011; 5(5):647-662.

2. Decramer M, Janssens W, Miravitlles M. Chronic obstructive pulmonary disease. Lancet. 2012;379(9823):1341-1351.

3. Singh V, Martinezclark P, Pascual M, Shaw ES, O'Neill WW. Cardiac biomarkers - the old and the new: a review. Coron Artery Dis. 2010; 21(4):244-256.

4. Røysland R, Kravdal G, Høiseth AD, et al. Cardiac troponin T levels and exercise stress testing in patients with suspected coronary artery disease: the Akershus Cardiac Examination (ACE) 1 study. Clin Sci (Lond). 2012;122(12):599-606.

5. Turer AT, Addo TA, Martin JL, et al. Myocardial ischemia induced by rapid atrial pacing causes troponin $\mathrm{T}$ release detectable by a high sensitive assay: insights from a coronary sinus sampling study. $J \mathrm{Am}$ Coll Cardiol. 2011;57(24):2398-2405.

6. Hickman PE, Potter JM, Aroney C, et al. Cardiac troponin may be released by ischemia alone, without necrosis. Clin Chim Acta. 2010; 411(5-6):318-323.

7. Brekke PH, Omland T, Holmedal SH, Smith P, Søyseth V. Troponin T elevation and long-term mortality after chronic obstructive pulmonary disease exacerbation. Eur Respir J. 2008;31(3):563-570.

8. Høiseth AD, Neukamm A, Karlsson BD, Omland T, Brekke PH, Søyseth V. Elevated high-sensitivity cardiac troponin T is associated with increased mortality after acute exacerbation of chronic obstructive pulmonary disease. Thorax. 2011;66(9):775-781.

9. Høiseth AD, Omland T, Hagve TA, Brekke PH, Søyseth V. Determinants of high-sensitivity cardiac troponin $\mathrm{T}$ during acute exacerbation of chronic obstructive pulmonary disease: a prospective cohort study. BMC Pulm Med. 2012;12:22.

10. Søyseth V, Bhatnagar R, Holmedahl NH, et al. Acute exacerbation of COPD is associated with fourfold elevation of cardiac troponin $\mathrm{T}$. Heart. 2013;99(2):122-126.

11. Neukamm AM, Høiseth AD, Hagve TA, Søyseth V, Omland T. Highsensitivity cardiac troponin $\mathrm{T}$ levels are increased in stable COPD. Heart. 2013;99(6):382-387.

12. Thurlbeck WM, Wright JL. Disease in the other organ. In: William Michael Thurlbeck, Joanne Lynne Wright, editors. Thurlbeck's Chronic Airflow Obstruction. 2nd ed. London, UK: BC Decker Inc; 1999.

13. Bhatt SP, Dransfield MT. Chronic obstructive pulmonary disease and cardiovascular disease. Transl Res. 2013;162(4):237-251.

14. [No authors listed]. Standardization of spirometry, 1994 update. American Thoracic Society. Am J Respir Crit Care Med. 1995;152(3): $1107-1136$.

15. Global Initiative for Chronic Obstructive Lung Disease. 2004 update: Workshop Report, Global Strategy for Diagnosis, Management and Prevention ofCOPD. Available from: http://www.goldcopd.org/. Accessed October 19, 2014.

16. Kamio K, Ishii T, Motegi T, et al. Decreased serum transforming growth factor- $\beta 1$ concentration with aging is associated with the severity of emphysema in chronic obstructive pulmonary disease. Geriatr Gerontol Int. 2013;13(4):1069-1075.

17. Hardie JA, Buist AS, Vollmer WM, Ellingsen I, Bakke PS, Mørkve O. Risk of over-diagnosis of COPD in asymptomatic elderly neversmokers. Eur Respir J. 2002;20(5):1117-1122.

18. Barnes PJ, Celli BR. Systemic manifestations and comorbidities of COPD. Eur Respir J. 2009;33(5):1165-1185.

19. Zervas E, Samitas K, Gaga M, et al. Inhaled corticosteroids in COPD: pros and cons. Curr Drug Targets. 2013;14(2):192-224.

20. Budweiser S, Jörres RA, Pfeifer M. Treatment of respiratory failure in COPD. Int $J$ Chron Obstruct Pulmon Dis. 2008;3(4): 605-618. 
21. deFilippi CR, de Lemos JA, Christenson RH, et al. Association of serial measures of cardiac troponin $\mathrm{T}$ using a sensitive assay with incident heart failure and cardiovascular mortality in older adults. JAMA. 2010;304(22):2494-2502.

22. Eggers KM, Al-Shakarchi J, Berglund L, et al. High-sensitive cardiac troponin $\mathrm{T}$ and its relations to cardiovascular risk factors, morbidity, and mortality in elderly men. Am Heart J. 2013;166(3):541-548.

23. Van Remoortel H, Hornikx M, Langer D, et al. Risk factors and comorbidities in the preclinical stages of chronic obstructive pulmonary disease. Am J Crit Care Med. 2014;189(1):30-38.

24. Lachin JM. Introduction to sample size determination and power analysis for clinical trials. Control Clin Trials. 1981;2(2):93-113.
25. Inami T, Seino Y, Otsuka T, et al. Links between sleep disordered breathing, coronary atherosclerotic burden, and cardiac biomarkers in patients with stable coronary artery disease. J Cardiol. 2012;60(3):180-186.

26. Randby A, Namtvedt SK, Einvik G, et al. Obstructive sleep apnea is associated with increased high-sensitivity cardiac troponin $\mathrm{T}$ levels. Chest. 2012;142(3):639-646.

27. Zamarrón C, García Paz V, Morete E, del Campo Matías F. Association of chronic obstructive pulmonary disease and obstructive sleep apnea consequences. Int J Chron Obstruct Pulmon Dis. 2008;3(4):671-682. 


\section{Supplementary materials Study design}

The study design is shown in Figure S1. The subjects were recruited from March 2005 to December 2013 at the Respiratory Care Clinic, which is a secondary referral facility affiliated with the Nippon Medical School in Tokyo, Japan. From a total of 548 subjects, 86 who either had or were at risk of chronic obstructive pulmonary disease (COPD) were selected as the patient group, and any patients with cardiac and/or pulmonary comorbidities after various examinations were carefully excluded (details in the following section on Exclusion criteria).

\section{Exclusion criteria}

Subjects with only COPD were examined in this study, excluding any cardiac and pulmonary comorbidities. Subjects suffering from
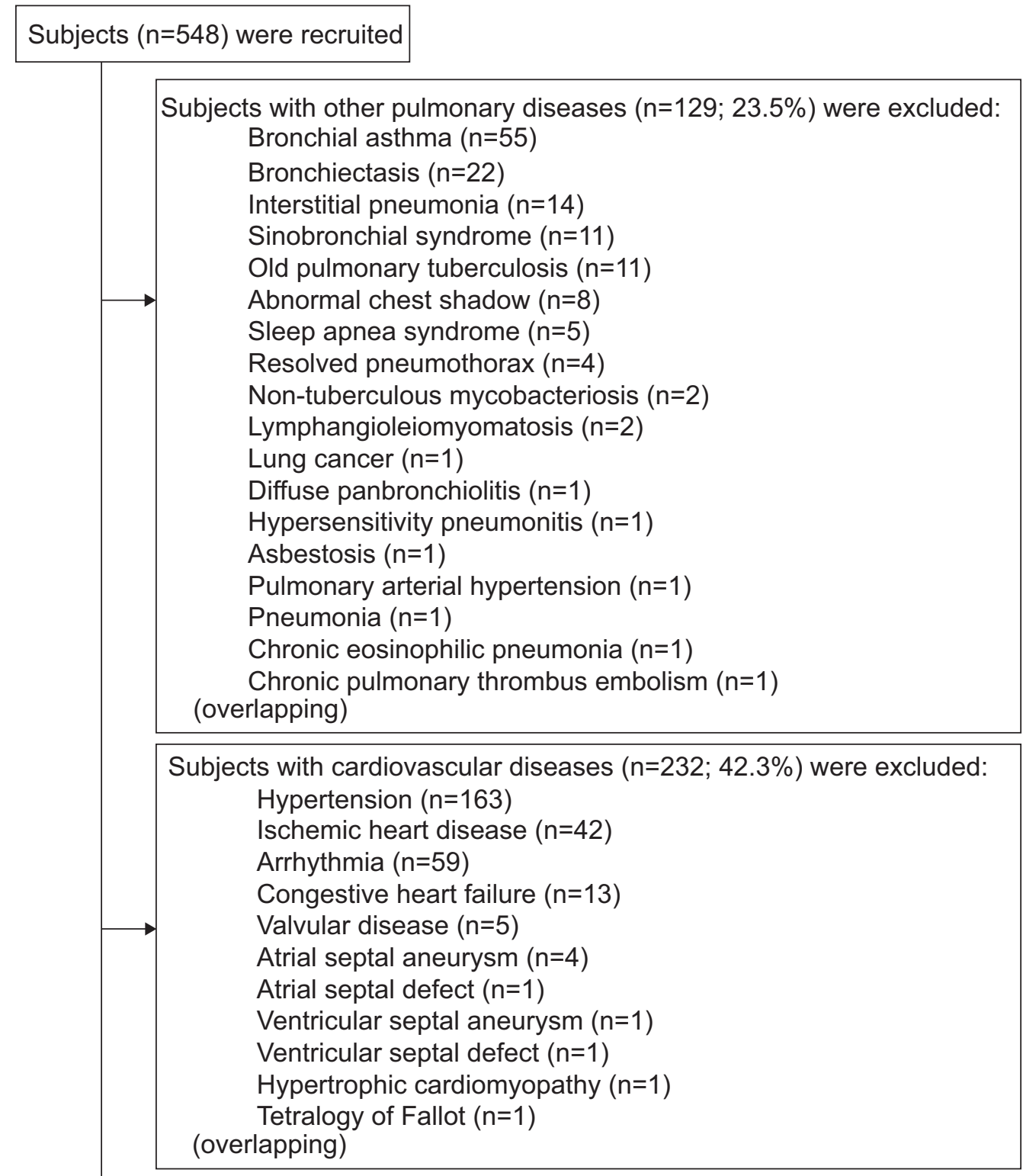

Subjects with current or ex-smoking habit $(n=187)$

Informed consent and enrollment

Subjects $(n=86)$

Figure SI Study flow chart. Subjects $(n=548)$ were recruited, and those with other pulmonary diseases ( $n=129$; eg, bronchial asthma and bronchiectasis) and/or any cardiovascular diseases $(n=232$; eg, hypertension and ischemic heart disease) were all excluded. Some subjects had multiple comorbidities. A total of 86 subjects were enrolled in the study. 
Table SI Multiple regression analysis for hs-cTnT levels (logged) in the group with chronic obstructive pulmonary disease

\begin{tabular}{|c|c|c|c|c|c|c|}
\hline Explanatory variable & B & $95 \% \mathrm{Cl}$ & & $\beta$ & $t$-value & $P$-value \\
\hline (Constant) & -2.931 & -3.549 & -2.314 & & -9.582 & 0.000 \\
\hline hs-CRP (logged) & 0.108 & 0.011 & 0.206 & 0.298 & 2.245 & 0.030 \\
\hline Age & 0.012 & 0.003 & 0.020 & 0.386 & 2.860 & 0.007 \\
\hline RVSP & 0.003 & -0.005 & 0.011 & 0.101 & 0.764 & 0.449 \\
\hline
\end{tabular}

Abbreviations: $B$, regression coefficient; $\mathrm{Cl}$, confidence interval; $\beta$, standardized regression coefficient; RVSP, right ventricular systolic pressure; hs-CRP, high-sensitivity C-reactive protein; hs-cTNT, high-sensitivity cardiac troponin T.

Table S2 Multiple regression analysis for hs-cTnT levels (logged) in the at-risk group

\begin{tabular}{|c|c|c|c|c|c|c|}
\hline Explanatory variable & B & $95 \% \mathrm{Cl}$ & & $\beta$ & $t$-value & $P$-value \\
\hline (Constant) & -2.867 & -3.489 & -2.245 & & -9.682 & 0.000 \\
\hline hs-CRP (logged) & 0.168 & 0.037 & 0.298 & 0.437 & 2.700 & 0.015 \\
\hline Age & 0.009 & 0.003 & 0.015 & 0.528 & 3.232 & 0.005 \\
\hline RVSP & 0.008 & -0.008 & 0.024 & 0.181 & 1.078 & 0.295 \\
\hline
\end{tabular}

Abbreviations: $B$, regression coefficient; $\mathrm{Cl}$, confidence interval; $\beta$, standardized regression coefficient; hs-CRP, high-sensitivity $C$-reactive protein; RVSP, right ventricular systolic pressure; hs-cTnT, high-sensitivity cardiac troponin T.

Table S3 Association between hs-cTnT levels (logged) and demographic and cardiopulmonary parameters in subjects with median or higher level of hs-TnT and in subjects with lower levels of hs-TnT

\begin{tabular}{|c|c|c|c|c|}
\hline & \multicolumn{2}{|c|}{$\begin{array}{l}\text { Hs-cTnT } \geq 0.007 \\
(n=4 I)\end{array}$} & \multicolumn{2}{|c|}{$\begin{array}{l}\text { Hs-cTnT }<0.007 \\
(n=45)\end{array}$} \\
\hline & $r$ & $P$-value & $r$ & $P$-value \\
\hline Age & 0.114 & 0.479 & 0.246 & 0.141 \\
\hline Smoking habit & 0.450 & 0.013 & 0.163 & 0.457 \\
\hline $\mathrm{BMI}$ & 0.179 & 0.264 & -0.053 & 0.756 \\
\hline FVC & -0.325 & 0.038 & -0.020 & 0.907 \\
\hline FEV $\%$ predicted (logged) & -0.132 & 0.410 & -0.099 & 0.562 \\
\hline \% DLco/VA (logged) & -0.287 & 0.069 & -0.142 & 0.403 \\
\hline $\mathrm{P}_{\mathrm{a}} \mathrm{O}_{2}$ & -0.182 & 0.304 & 0.060 & 0.760 \\
\hline WBC & 0.042 & 0.796 & 0.252 & 0.132 \\
\hline BNP & 0.164 & 0.333 & 0.206 & 0.222 \\
\hline hs-CRP (logged) & 0.418 & 0.007 & 0.234 & 0.163 \\
\hline IVS Td & 0.012 & 0.939 & 0.004 & 0.983 \\
\hline PW Td & 0.082 & 0.612 & 0.037 & 0.830 \\
\hline LAD & 0.036 & 0.825 & 0.279 & 0.095 \\
\hline LVEDV & -0.028 & 0.864 & 0.161 & $0.34 I$ \\
\hline EF & -0.012 & 0.939 & -0.076 & 0.654 \\
\hline $\mathrm{CO}$ & 0.010 & 0.954 & $0.34 I$ & 0.039 \\
\hline RVSP & 0.327 & 0.051 & -0.111 & 0.951 \\
\hline Walking distance (logged) & -0.310 & 0.048 & -0.207 & 0.219 \\
\hline$\Delta \mathrm{SpO}_{2}$ & 0.049 & 0.763 & 0.100 & 0.558 \\
\hline LAA\% for the upper lung field & 0.184 & 0.249 & -0.025 & 0.884 \\
\hline LAA\% for the lower lung field & -0.010 & 0.950 & -0.013 & 0.939 \\
\hline
\end{tabular}

Abbreviations: BMI, body mass index; FVC, forced vital capacity; $\mathrm{FEV}_{\text {, }}$, forced expiratory volume in I second; DLco/VA, diffusing capacity of the lung for carbon monoxide/ alveolar volume; $\mathrm{P}_{2} \mathrm{O}_{2}$, partial pressure of oxygen; WBC, white blood cells; BNP, brain natriuretic peptide; hs-CRP, high-sensitivity C-reactive protein; hs-cTnT, highsensitivity cardiac troponin T; IVS Td, thickness of interventricular septum in diastole; PW Td, thickness of posterior wall in diastole; LAD, left atrial dimension; LVEDV, left ventricular end-diastolic volume; $\mathrm{EF}$, ejection fraction; $\mathrm{CO}$, cardiac output; RVSP, right ventricular systolic pressure; $\triangle \mathrm{SPO} \mathrm{O}_{2}$, delta saturation of pulse oximetry oxygen; LAA, low attenuation area. 
Table S4 Multiple regression analysis for hs-cTnT levels (logged) in subjects with median or higher levels of hs-TnT (stepwise estimation model)

\begin{tabular}{|c|c|c|c|c|c|c|}
\hline Explanatory variable & B & $95 \% \mathrm{Cl}$ & & $\beta$ & $t$-value & $P$-value \\
\hline (Constant) & -1.830 & -1.930 & -1.731 & & -37.783 & 0.000 \\
\hline hs-CRP (logged) & 0.157 & $0.07 \mid$ & 0.243 & 0.586 & 3.761 & 0.001 \\
\hline
\end{tabular}

Abbreviations: $B$, regression coefficient; $\mathrm{Cl}$, confidence interval; $\beta$, standardized regression coefficient; hs-CRP, high-sensitivity $C$-reactive protein; hs-cTnT, high-sensitivity cardiac troponin $\mathrm{T}$.

any one of these pulmonary and/or cardiovascular diseases were excluded: bronchial asthma, bronchiectasis, interstitial pneumonia, sinobronchial syndrome, active or old pulmonary tuberculosis, non-tuberculous mycobacteriosis, abnormal chest shadow suggestive of a lung tumor, resolved pneumothorax, lymphangioleiomyomatosis, lung cancer, diffuse panbronchiolitis, hypersensitivity pneumonitis, asbestosis, pulmonary hypertension, pneumonia, chronic eosinophilic pneumonia, chronic pulmonary thromboembolism, hypertension, ischemic heart disease, any type of arrhythmia, congestive heart failure, atrial septal aneurysm, ventricular septal aneurysm, hypertrophic cardiomyopathy, valvular disease, atrial septal defect, ventricular septal defect, tetralogy of Fallot, and genetic $\alpha 1$ anti-trypsin deficiency. Thus, careful attention was paid to exclude all these cases with cardiopulmonary comorbidities in order to focus on subjects with only COPD or those with a lifelong smoking history who exhibited clinical symptoms similar to COPD, whom we considered at-risk subjects.

\section{Pulmonary function tests}

The tests were performed by well-trained technicians according to the American Thoracic Society guidelines ${ }^{1}$ using specialized equipment for lung function testing with computer processing (Chestac 55; Chest Co., Tokyo, Japan). The standards of the Japanese Respiratory Society ${ }^{2}$ were used as the reference values for post-bronchodilator forced expiratory volume in 1 second and forced vital capacity.

\section{High-resolution computed tomography}

We performed helical high-resolution computed tomography scans at $1.25 \mathrm{~mm}$ collimation, 0.8 second scan time (rotation time), $120 \mathrm{kV}$, and 100-600 mA using a Light Speed Pro16 CT Scanner (GE Co., Tokyo, Japan).

\section{Echocardiography}

The same experienced echocardiographer performed the procedure on all subjects. The examination was recorded in the standard parasternal and apical views during normal breathing at end-expiration. All measurements were obtained according to the standards of the American Society of Echocardiography. ${ }^{3}$ The left ventricular internal end-diastolic and end-systolic diameters were measured over five consecutive cycles. Systolic pulmonary artery pressure was calculated using the modified Bernoulli equation with an estimated right atrial pressure of $10 \mathrm{mmHg}$.

\section{Multiple regression analysis for hs-cTnT levels (logged) in the COPD group and at-risk group}

We determined whether this multivariate model for highsensitivity cardiac troponin T (hs-cTnT) would be accurate for both a subgroup of COPD and at-risk subjects. High-sensitivity C-reactive protein and age, but not right ventricular systolic pressure, were associated with hs-cTnT using this model for these two subgroups (Tables S1 and S2).

\section{Association between hs-cTnT levels (logged) and demographic and cardiopulmonary parameters in subjects with median or higher hs-TnT levels and in subjects with lower hs-TnT levels}

The hs-cTnT level was low in the entire population. Thus, we also performed simple correlation analyses between hs-cTnT and clinical parameters (Table S3), and performed stepwise regression analysis on logged hs-cTnT values (Table S4) again in the subjects whose hs-cTnT was greater than the median (0.007). Although the population was relatively small in this analysis, we were still able to observe an association between hs-cTnT and C-reactive protein.

\section{References}

1. Global Initiative for Chronic Obstructive Lung Disease Executive Committee. 2004 update: Workshop Report, Global strategy for the diagnosis, management, and prevention of chronic obstructive pulmonary disease. Medical Communications Resources, Inc. Updated 2004. Available from: http://www.goldcopd.org/. Accessed October 19, 2014.

2. Japanese Respiratory Society. [The predicted values of spirometry and arterial blood gas analysis in Japanese]. J Jpn Respir Soc. 2001;39:1-17. Japanese.

3. Cheitlin MD, Armstrong WF, Aurigemma GP, et al. American College of Cardiology, American Heart Association, American Society of Echocardiography. ACC/AHA/ASE 2003 guideline update for the clinical application of echocardiography: summary article: a report of the American College of Cardiology/American Heart Association Task Force on Practice Guidelines (ACC/AHA/ASE Committee to Update the 1997 Guidelines for the Clinical Application of Echocardiography). Circulation. 2003;108(9):1146-1162. 


\section{Publish your work in this journal}

The International Journal of COPD is an international, peer-reviewed journal of therapeutics and pharmacology focusing on concise rapid reporting of clinical studies and reviews in COPD. Special focus is given to the pathophysiological processes underlying the disease, intervention programs, patient focused education, and self management protocols.

This journal is indexed on PubMed Central, MedLine and CAS. The manuscript management system is completely online and includes a very quick and fair peer-review system, which is all easy to use. Visit http://www.dovepress.com/testimonials.php to read real quotes from published authors.

Submit your manuscript here: http://www.dovepress.com/international-journal-of-chronic-obstructive-pulmonary-disease-journal 